\title{
L'intercompréhension et la grammaire comparée au service d'un apprentissage plurilingue : MultiGram et Roma·Net
}

\author{
Annick Englebert, Sabina Gola
}

\begin{abstract}
Les deux plates-formes MultiGram (http ://multigram.ulb.ac.be) et Roma.Net (http ://romanet.ulb.be) ont été conçues comme outils complémentaires dans le cadre de l'enseignement/apprentissage des langues. La première réunit six langues, trois germaniques (l'allemand, l'anglais, le néerlandais) et trois romanes (l'espagnol, le français, l'italien); la deuxième, cinq langues romanes (espagnol, français, italien, portugais et roumain). Elles ont une structure totalement différente et prennent en compte des aspects distincts des langues : l'une a comme point de départ la grammaire contrastive, l'autre plutôt l'intercompréhension entre les langues romanes; l'une mise également sur l'approche communicative, l'autre sur la dimension culturelle. Malgré ces différences substantielles, les objectifs poursuivis sont les mêmes : encourager les apprenants à se servir de leurs expériences linguistiques tant dans leur langue maternelle que dans d'autres langues étudiées auparavant, ainsi qu'à poursuivre leur apprentissage de façon autonome. Ces deux plates-formes s'adressent sans doute à un public déjà plurilingue désireux d'ajouter d'autres langues à son bagage linguistique, mais aussi à un apprenant monolingue qui a envie d'apprendre une nouvelle langue tout seul ou approfondir ses connaissances dans une langue qu'il connaît déjà. En effet, MultiGram et Roma·Net peuvent être utilisés comme des outils traditionnels d'apprentissage des langues (une langue à la fois). Grâce à leur flexibilité, due en partie à la technologie, les deux plates-formes permettent aux enseignants et aux apprenants de créer des parcours d'apprentissage personnalisés qui tiennent compte du répertoire linguistique des utilisateurs. L'encouragement à la participation active des étudiants à leur apprentissage est aussi une caractéristique de ces outils. Dans notre communication, dans un premier temps, nous allons intégrer MultiGram et Roma·Net dans le cadre des objectifs du plurilinguisme promus dans le Cadre Européen Commun de Référence pour les langues, en évoquant également le concept d'intercompréhension. Puis, nous allons approfondir quelques concepts fondamentaux sur lesquels se basent les plates-formes, comme le rôle de la langue maternelle dans l'apprentissage des langues, le rôle des autres langues connues sur l'apprentissage d'une nouvelle langue, l'importance de la motivation dans l'apprentissage. Sur la base de notre expérience personnelle, nous montrerons aussi quelques scénarios pédagogiques d'apprentissage.
\end{abstract}

Mots-clés : grammaire contrastive, approche communicative, intercompréhension, plurilinguisme, didactique, langues romanes, langues germaniques, apprentissage en autonomie

Abstract : MultiGram (http://multigram.ulb.ac.be) and Roma.Net (http://romanet.ulb.be)
have been designed as complementary tools in the context of language teaching / learning.
The first brings together six languages, three Germanic languages (German, English, Dutch)
and three Romance languages (Spanish, French, Italian); the second five Romance languages
(Spanish, French, Italian, Portuguese and Romanian). They have a totally different structure
and take into account different aspects of languages: one is based on contrastive grammar, the
other on intercomprehension between Romance languages; one also focuses on the commu- 
nicative approach, the other on the cultural dimension. Despite these substantial differences, the objectives are the same: to encourage learners to use their linguistic experiences both in their mother tongue and in other languages studied previously, and to pursue their learning independently. These two platforms are no doubt aimed at an already multilingual public wishing to add other languages to its linguistic background, but also to a monolingual learner who wants to learn a new language by himself or to deepen his knowledge in one language, he already knows. Indeed, MultiGram and RomaNet can be used as traditional tools for learning languages (one language at a time). Due to their flexibility, due in part to technology, both platforms allow teachers and learners to create personalized learning paths that take into account the users' language repertoire. Encouraging the active participation of students in their learning is also a characteristic of these tools. In our communication, as a first step we will integrate MultiGram and RomaNet within the framework of the plurilingualism objectives promoted in the Common European Framework of Reference for Languages, while also mentioning the concept of intercomprehension. Then, we will deepen some fundamental concepts on which the platforms are based, such as the role of the mother tongue in language learning, the role of other languages known on learning a new language, the importance of motivation in learning ... Based on our personal experience, we will also show some learning scenarios.

Key words: comparative grammar, communicative approach, intercomprehension, multilingualism, didactics, Romance languages, Germanic languages, autonomous learning

\section{Introduction}

«L'anglais, langue ennemie ou amie?» Tel est le titre d'un chapitre de la section «L'éveil au plurilinguisme» dans l'ouvrage que Sandrine Caddéo et Marie-Christine Jamet ont consacré à L'intercompréhension : une nouvelle approche pour l'enseignement des langues (Hachette, 2013). C'est une question que les enseignants ${ }^{1}$ des langues autres que l'anglais se posent de manière récurrente, spécialement dans le monde universitaire européen qui est caractérisé par une politique linguistique très variée, mais sûrement favorable à l'enseignement prioritaire de l'anglais. Dans leur ouvrage, Caddéo et Jamet passent en revue les discours les plus communs en faveur ou en défaveur de l'utilisation de l'anglais comme langue de communication, qu'ils soient de nature politique, commerciale ou économique. Il ressort de leur examen que, malgré son statut de lingua franca, dans le monde professionnel, on ne se contente plus du «Global English », c'est-à-dire «d'un anglais utilitaire détaché des pratiques culturelles dans lesquelles une langue s'inscrit » (Caddéo, Jamet, 2013, p. 18).

La question qui se pose alors aux enseignants est celle de la voie à emprunter pour convaincre les étudiants, futur citoyens européens, d'apprendre d'autres langues et de ne pas se contenter de la connaissance d'une langue de communication artificielle - il n'est peut-être pas inutile de rappeler en effet que le BASIC

${ }^{1}$ L'utilisation du genre masculin dans le présent texte a pour simple but d'alléger le style. Elle ne marque aucune discrimination à l'égard des femmes. 
English ('British American Scientific International Commercial') avait été mis au point pour rendre l'anglais rapidement assimilable par les populations issues de la colonisation.

Une réponse à cette question pourrait bien venir de l'anglais lui-même. L'anglais présente en effet cette caractéristique que, tout en appartenant, typologiquement, à la famille des langues germaniques, son histoire l'a amené à emprunter de nombreux traits, tant lexicaux que syntaxiques, au français et à puiser directement dans le latin pour enrichir son lexique, de sorte qu'il présente aujourd'hui de nombreuses affinités avec le français, ainsi que plus généralement avec les langues romanes - dans la famille desquelles certains linguistes, mettant en avant des arguments plus « organiques » que génétiques et typologiques, vont jusqu'à le ranger. Il en résulte que l'anglais et le français peuvent jouer, chacun à leur tour, et en fonction des situations et de la langue maternelle des locuteurs, le rôle de passerelle de leur propre famille vers la famille voisine.

L'approche par l'intercompréhension, basée sur les similitudes phonétiques, morphologiques et lexicales entre les langues d'une même famille linguistique, apparait alors comme une réponse à la question des stratégies à utiliser pour que les apprenants s'ouvrent à d'autres langues que l'anglais. Nous avons ainsi pu observer que les étudiants qui étudient l'anglais à l'université, lorsqu'ils sont placés devant un fragment de texte en français du Moyen Âge ${ }^{2}$, sont, dans un premier temps, amusés d'y découvrir un verbe remembrer qui signifie 'se souvenir' ou un verbe recorder 'graver dans son cur/sa mémoire'; dans un second temps, ils se montrent souvent plus performants dans l'exercice de traduction du français médiéval vers le français moderne que les étudiants qui n'étudient «que» le français, parce qu'ils puisent ainsi aussi bien dans leur répertoire du français, L1, que dans leur répertoire de l'anglais, L2, pour comprendre le texte en L3 qu'ils ont sous les yeux (cf. Gyroux, 2016); ils se servent de leurs capacités de déduction, en développent de nouvelles (un défi pour les enseignants et pour les apprenants) et progressent simultanément dans l'apprentissage de l'anglais comme dans leur connaissance du français. Par l'intercompréhension, ils s'ouvrent ainsi à la langue française et, s'ils sont un peu curieux, aux autres langues romanes qu'ils peuvent connaitre, par apprentissage scolaire ${ }^{3}$, par leur milieu familial ou tout simplement parce qu'ils ont été en contact avec elles lors de voyages.

L'anglais devient alors une langue attractive, non pas en raison de la position hégémonique qu'elle occupe, mais en raison de la place qu'elle occupe au sein

\footnotetext{
2 Ce fut le cas pour les étudiants d'anglais du département de Langues et Lettres de l'ULB de 2011 à 2013.

${ }^{3}$ Dans le système universitaire belge, l'apprentissage de l'anglais L2 est toujours couplé à l'apprentissage d'une L3, de la même famille ou d'une autre famille.
}

$6 \quad$ Studie/Study 
d'un ensemble et, dans une perspective de plurilinguisme, des passerelles qu'elle permet de jeter entre les langues (cf. Caddéo et Jamet, 2013, p. 19).

\section{Plurilinguisme et didactique}

Depuis désormais deux décennies, les concepts de compétence plurilingue et pluriculturelle se sont frayé un chemin dans le domaine de l'apprentissage et de l'enseignement des langues (Moore \& Castellotti 2008, Beacco et al. 2010, Gajo 2019). Est-ce que ces concepts ont influencé la didactique des langues? Est-ce que l'adoption d'une "approche singulière », c'est-à-dire l'étude d'une langue et d'une culture de façon isolée, est encore la pratique didactique la plus suivie ou est-ce que ce sont les «approches plurielles » - l'étude de différentes langues et cultures à la fois - qui prévalent dans les établissements scolaires? Telles sont les questions que l’on est amené à se poser aujourd'hui (Troncy 2014).

Les pratiques didactiques ont beaucoup changé dernièrement, grâce notamment aux recherches menées en psycholinguistique sur l'acquisition d'une seconde langue, qui ont permis de mettre en évidence l'existence d'influences réciproques dans l'apprentissage de différentes langues (Troncy 2014). Ceci a amené les enseignants à s'ouvrir à une didactique des langues intégrée, à l'utilisation de l'intercompréhension (Carrasco 2008, Caddéo, Jamet 2013, Sheeren 2016) ou à la pratique de l'éveil aux langues.

Même si les nouvelles théories sur l'apprentissage des langues peuvent inspirer des modèles didactiques nouveaux, dans la réalité, l'enseignement des langues selon des approches plurielles est encore très limité et c'est surtout dans les établissements scolaires qui choisissent de participer à des projets européens qu'une réflexion accompagnée d'activités pratiques ciblées est menée (Gajo 2019).

Toutefois, en dehors des structures d'enseignement, la situation est très différente. Les individus eux-mêmes, et en nombre de plus en plus élevé, pour les raisons les plus variées, acquièrent plusieurs langues à la fois, ce qui leur permet d'arriver à exploiter leur bagage langagier dans les situations les plus diverses et suivant des modalités différentes selon les contextes communicatifs.

\section{MultiGram}

C'est pour encourager les enseignants à adopter une approche «translinguistique» (Troncy 2016) et également pour offrir un outil de support à tous ceux qui, par nécessité ou curiosité, s'attèlent à l'étude des langues, encadrée ou en autonomie, que MultiGram a vu le jour.

Développée dès 2012 par une petite équipe d'enseignants de langues, MultiGram (https ://multigram.ulb.ac.be) est une plate-forme multilingue libre d'accès qui 
offre à l'apprenant et à l'enseignant un dispositif d'acquisition d'une ou plusieurs langues-cibles - dans l'état actuel de son développement, trois langues germaniques (allemand, anglais et néerlandais) et trois langues romanes (espagnol, français et italien).

Cette plate-forme se présente, pour chaque langue décrite, sous la forme de fiches indépendantes, reliées entre elles par des hyperliens qui permettent de naviguer à l'intérieur d'une langue, entre des fiches apparentées par le sujet qu'elles traitent. Ces liens sont réversibles, de sorte que l'utilisateur peut aisément revenir à son point de départ; par exemple, la fiche consacrée aux liens de parenté renvoie à la fiche sur les adjectifs possessifs et la fiche consacrée aux adjectifs possessifs renvoie aux liens de parenté.

Une zone de navigation spécifique signale expressément les corrélations entre langues lorsqu'elles existent et permet de passer aisément d'une langue à l'autre, quand le sujet traité dans une fiche est pertinent dans plusieurs langues.

\section{2.a Structuration}

Les fiches sont structurées en deux portails, un portail grammatical, où elles s'organisent selon les catégories grammaticales traditionnelles - le groupe nominal, le groupe verbal, la phrase ... - et un portail communicationnel, où elles s'organisent selon les situations de communication auxquelles un locuteur peut être confronté : comment exprimer l'accord ou le désaccord, le but, la certitude, la durée d'une action, l'hypothèse ...; comment employer tel mot ou telle expression (par exemple, pour le français, d'accord, s'il vous plait, pardon, à la limite ... ou pour l'anglais whole all, as like). L'organisation du portail communicationnel renvoie en outre aux différents niveaux du cadre européen de référence pour les langues (CECRL 2001, 2018).

\section{2.b Multilinguisme}

Le caractère multilingue de MultiGram est exclusivement dans les langues qu'elle cible.

En effet, quelle que soit la langue ciblée par la description, la langue de rédaction et de comparaison des fiches est uniformément le français - un choix rédactionnel motivé par le fait que l'outil a été développé au sein d'une université (l'Université Libre de Bruxelles) dont le français est la principale langue d'enseignement.

Mais si la langue de rédaction est commune à toutes les fiches, la structure du double portail est spécifique à chaque langue décrite. En d'autres termes, même si la langue française sert de référence pour la description de toutes les langues, les fiches qui décrivent les autres langues ne sont pas conçues comme des tra- 
ductions des fiches qui décrivent le français : la partie de la plate-forme vouée à l'anglais ne compte aucune fiche relative à l'accord du participe passé, difficulté grammaticale incontournable de la langue française; à l'inverse, la partie de la plate-forme vouée à l'allemand contient des fiches relatives à la déclinaison qui ne doivent rien à la description du français.

Si traduction il y a, c'est uniquement au niveau des exemples illustratifs des faits de langue décrits, des exemples qui sont toujours donnés dans la langue-cible, mais accompagnés d'une traduction en français.

\section{2.c Parcours d'apprentissage}

L'un des aspects les plus novateurs de MultiGram est dans sa flexibilité.

En effet, même si la plate-forme a été dotée de différents cadres - table des matières d'une grammaire traditionnelle, CECRL - qui peuvent guider l'utilisateur dans un apprentissage linéaire et progressif, somme toute traditionnel, de la langue, elle permet aussi à l'utilisateur de se créer des parcours d'apprentissage personnels, en fonction de ce qu'il sait déjà ou de ce qu'il ne sait pas encore d'une langue, d'accéder à tout moment aux points de grammaire qu'il a besoin de réviser, quelle que soit la voie d'entrée qu'il a empruntée. Elle lui permet de « rebondir» de fiche en fiche en fonction des liens qui y figurent ou d'utiliser le moteur de recherche interne à la plate-forme si le contenu d'une fiche ne fournit pas la réponse à la question qu'il se pose.

MultiGram permet ainsi à l'utilisateur de se créer des parcours inventifs, se laissant guider par la simple curiosité, découvrant des similitudes entre langues qu'il ne soupçonnait pas nécessairement au départ. ${ }^{4}$

\section{2.d Évolution}

La plate-forme MultiGram se caractérise enfin par le fait que ses contenus ne sont pas figés. D'une part la description de chaque langue est susceptible de continuer de s'enrichir chaque jour - les descriptions des différentes langues n'attestent d'ailleurs pas toutes d'un même état d'avancement du projet. D'autre part, elle reste ouverte à la description d'autres langues, représentant les mêmes familles romane et germanique ou ouvrant vers d'autres familles. Enfin, l'enrichissement du contenu pour chaque langue n'est pas réservé uniquement à des spécialistes en langues ou en didactique; les étudiants, sous la supervision de leurs enseignants, peuvent également y contribuer et devenir les vrais acteurs de leurs apprentissages.

\footnotetext{
${ }^{4}$ Cette manière «moins traditionnelle» d'aborder la grammaire des langues est citée par les étudiants de l’Université Libre de Bruxelles comme un des atouts de l'outil.
} 


\section{Roma·Net}

Née en mars 2019, Roma-Net (http ://romanet.ulb.be) est une plate-forme libre d'accès conçue dans un esprit très différent de MultiGram, même si elle la rejoint dans sa volonté de répondre à de nouvelles manières d'appréhender les langues.

Cette autre plate-forme est en effet entièrement vouée à une approche contrastive des langues et cultures romanes - il ne s'agit donc pas d'un outil qui se positionne par rapport à l'anglais, même s'il n'est pas dénué d'intérêt pour un anglophone ou pour un apprenant d'anglais, ne serait-ce que par les liens qu'entretient l'anglais avec les langues romanes, comme nous le rappelions dans notre introduction.

Bien que jeune, cette plate-forme est le fruit d'une collaboration de plusieurs années : elle est la trace pérenne d'ateliers organisés annuellement à l'Université Libre de Bruxelles, à l'intention des élèves des classes terminales et qui ont pour objectif de leur faire découvrir la richesse des langues et cultures romanes et les passerelles que l'on peut jeter entre les différentes langues romanes et entre les différentes cultures romanes. Il s'agit chaque fois de montrer comment une thématique imposée - la liberté, la diversité, le conflit, l'émotion ... - s'exprime dans les différentes langues romanes et leurs cultures. Les animations, très courtes (45 minutes) et au rythme soutenu, mettent surtout en avant les sonorités (lecture d'un même texte dans les différentes langues romanes que les élèves doivent tenter d'identifier) et le caractère idiomatique de certaines expressions, mais les participants reçoivent un dossier complet développant en outre les aspects grammaticaux, lexicaux et culturels des différentes langues. C'est de ces dossiers qu'est né le projet de plate-forme Roma-Net.

Les langues romanes décrites dans Roma-Net sont le français, l'italien, l'espagnol, le portugais et le roumain. Quelques informations relatives au latin ont été intégrées, mais elles ne sont pas systématisées, de même que les évocations des états anciens des langues romanes.

Comme MultiGram, Roma-Net est constituée de fiches, mais contrairement à MultiGram, l'approche contrastive étant ici systématisée, la présence d'une fiche pour une langue appelle aussi celle d'une fiche équivalente pour les autres langues en tout cas, au minimum pour le français, l'italien, l'espagnol, le portugais et le roumain.

Contrairement à MultiGram aussi, dont la ligne se veut résolument sobre et épurée, Roma-Net joue beaucoup sur son graphisme, chaque langue étant à la fois symbolisée par une couleur - le français est orange, l'italien est azur, l'espagnol est pomme ... - et représentée par un personnage porte-parole - Julie la francophone, Csar le latinophone, Blanca l'hispanophone, Giuseppe l'italophone ... 


\section{3.a Multilinguisme}

Comme dans MultiGram, le français est la langue de rédaction de toutes les pages de Roma-Net - et à ce stade la seule langue de rédaction. Ce choix connait les mêmes motivations que le choix rédactionnel de MultiGram, même si dans le cas de Roma-Net, il n'est pas exclu, en théorie, que la plateforme soit traduite dans les différentes langues romanes qu'elle décrit. ${ }^{5}$

\section{3.b Flexibilité}

L'un des principaux atouts de la plate-forme est ici encore dans sa flexibilité, qui se manifeste à travers la diversité des voies d'entrée. En effet, alors qu'une entrée par la table des matières d'un dossier, téléchargeable et imprimable, invite à un parcours linéaire et ordonné dans une thématique, la navigation sur internet autorise des parcours multiples et individualisés, qui se dessinent au gré des «clics» de l'utilisateur : pour l'un ce sera un parcours linéaire, pour l'autre un parcours bondissant d'un mot-clé à un autre, pour un autre, ce sera un va-et-vient entre deux langues, etc.

La plate-forme Roma-Net connait essentiellement deux portes d'entrées principales : l'entrée par les thèmes et l'entrée par les langues.

La voie d'entrée linguistique ouvre sur les champs du lexique, de la grammaire, des expressions idiomatiques; la voie d'entrée culturelle ouvre sur les champs de la littérature, de la chanson, de la peinture ... mais aussi sur celui des comportements sociaux ou encore celui des symboles nationaux; la voie d'entrée historique ouvre sur le champ des textes fondateurs des langues romanes.

Pour tous ces champs d'exploration, le choix est laissé à l'utilisation d'accéder aux informations et aux descriptions soit pour une seule langue romane, soit dans une perspective comparative, pan-romane. Certains champs de Roma-Net ne sont d'ailleurs explorés que dans cette perspective pan-romane : les cartes géolinguistiques et les textes «universels» (comme la Déclaration des droits de l'homme ou les grands textes des littératures romanes : Pinocchio, Le petit Prince ...).

\section{3.c Parcours d'utilisateurs}

Quelle que soit la porte d'entrée que l'utilisateur choisit, quel que soit le champ par lequel il commence son parcours, il a à partir de chacune des pages le loisir de poursuivre sur la même voie ou de bifurquer. Ainsi un utilisateur qui accède à Roma-Net avec pour objectif de s'informer sur un point de grammaire française

\footnotetext{
${ }^{5}$ L'ampleur du travail que cela représenterait nécessite des moyens que nous n’avons pas, aussi ce projet reste-t-il pour le moment à l'état de souhait et d'aspiration.
} 
peut très bien être attiré par l'approche différentielle et aller consulter ce que devient le même point de grammaire en roumain ou en espagnol ou encore dans quelle situation de communication ce point de grammaire entre en jeu. Tout est fonction des objectifs que l'on donne à son parcours et de son degré de curiosité.

\section{3.d Évolution}

Tout comme MultiGram, Roma-Net est une plate-forme collaborative, en constante évolution. Une version interactive, incluant des exercices ${ }^{6}$, est en cours de développement.

Grâce à la collaboration des étudiants de l'Université Libre de Bruxelles, elle devrait prochainement intégrer des informations sur le catalan, ainsi que sur certains dialectes romans - le sarde, le picard ... - et pourrait donner aux langues romanes minoritaires un «second souffle» (Sheeren, 2016).

\section{Conclusion}

Dans notre société en pleine mutation, enseigner les langues devient presque une gageure. Le profil des étudiants accueillis dans les départements de langue des universités a changé. Face à ces changements, nous avons fait le choix de développer des outils répondant davantage à ces nouveaux profils, c'est-à-dire à de nouvelles attentes et de nouveaux objectifs, mais aussi à de nouvelles manières d'apprendre et d'enseigner les langues.

En réponse à ces mutations, nous avons choisi de mettre en place deux outils, sous la forme de plates-formes multilingues, permettant aux étudiants comme aux enseignants de gérer plusieurs langues à la fois, sans imposer toutefois de parcours multilingue ou comparatiste dans l'apprentissage des langues.

L'enseignement et l'apprentissage d'une langue en elle-même et pour elle-même par le biais de ces deux outils demeurent certes possibles, mais le parcoursdécouverte dans des langues méconnues, l'ouverture, par les langues, à d'autres cultures, la réappropriation, par l'approche contrastive, de l'image que les locuteurs se font de leur langue maternelle ... y sont autant d'alternatives offertes à une approche "unidirectionnelle » de l'enseignement et de l'apprentissage des langues qui n'a plus les faveurs.

La souplesse d'utilisation et l'accès libre en sont des atouts majeurs, reconnus par l'ensemble des utilisateurs, qui soulignent aussi bien le fait que les commentaires

\footnotetext{
${ }^{6}$ Si l'on en juge par les souhaits exprimés par les étudiants utilisateurs de MultiGram à l'Université Libre de Bruxelles, l'existence d'exercices intégrés à la plate-forme serait un atout supplémentaire de celle-ci.
} 
soient formulés dans une même langue quelle que soit la langue ciblée que l'utilité d'exemples assortis de traductions'.

Roma·Net est un outil trop récent pour autoriser une prise de recul : pour autant qu'on puisse s'y fier après un peu plus d'un an d'existence, les statistiques révèlent d'une part que les utilisateurs de la plate-forme sont rapidement fidélisés, d'autre part que les pages consacrées à la linguistique sont davantage visitées que les autres, enfin que les pages de toutes les langues présentent le même taux de fréquentation. L'analyse statistique de MultiGram, plus révélatrice parce que bénéficiant de davantage de recul, montre d'une part que la plateforme est fréquentée par un public international, débordant largement le public étudiant pour lequel elle a été conçue, d'autre part que la page d'accueil d'une langue n'est pas la voie d'accès privilégiée par les utilisateurs de la plateforme, qui usent de tous les moyens technologiques à leur disposition (marque-pages, moteurs de recherche, hyperliens ...) pour en consulter les différentes fiches. MultiGram apparait ainsi comme une réponse appropriée à de nouveaux besoins et de nouveaux défis. Une réponse parmi d'autres ...

\section{Références bibliographiques}

BEACCO, J.-C. ET AL. (2016). Guide pour le développement et la mise en uvre de curriculums pour une éducation plurilingue et interculturelle. Conseil de l'Europe. En ligne : https ://rm.coe.int/ CoERMPublicCommonSearchServices/DisplayDCTMContent ?documentId=09000016806ae64a.

CADDÉO, S., \& JAMET, M.-C. (2013). L'intercompréhension : une autre approche pour l'enseignement des langues. Paris, Hachette (Collection «F»).

CARMEl, M. C., BiER, A., \& BALlARIN, E. (Eds.) (2018). La didattica delle lingue nel nuovo millennio. Le sfide dell'internazionalizzazione. Studi e ricerche 13.

Carrasco Perea, E., Degache, D., \& Pishva, Y. (2008). Intégrer l'intercompréhension à l'université, Les Langues modernes. L'intercompréhension. Travaux en intercompréhension : conceptions, outils et démarches pour la formation en langues, p. 62-74. En ligne : https ://www.researchgate.net/ publication/299461252_Integrer_1\%27intercomprehension_a_1\%27universite

ConsEIL DE L'EuRope (2001). Cadre europeen commun de reference pour les langues : Apprendre, Enseigner, Évaluer (CECRL). En ligne : https ://rm.coe.int/16802fc3a8.

CONSEIL DE l'EuRope (2018), Cadre Commun de Référence pour les langues : apprendre, enseigner, évaluer. En ligne : https ://rm.coe.int/cecr-volume-complementaire-avec-de-nouveaux-descripteurs/ $16807875 \mathrm{~d} 5$.

CONSEIL DE L'EuROPE (2011-2019). Cadre de référence pour les approches plurielles des langues et des cultures (CARAP). En ligne : https ://carap.ecml.at.

EscudÉ, P., \& JANIN, P. (2010). Le point sur l'intercompréhension, clé du plurilinguisme. Paris, CLE International.

GAJo, L. (2019). Didactique des langues et didactique du plurilinguisme : continuités, complémentarités, ruptures. In GolA, S., Pierrard, M., Tops, E., VAN RAEMdOnCK, D. (Eds.) Enseigner et apprendre les

\footnotetext{
${ }^{7}$ Les traductions des exemples compensent l'absence des listes de vocabulaire ou de dictionnaires au sein de la plate-forme, les utilisateurs pouvant se composer leurs propres listes de vocabulaire au gré des exemples qu'ils trouvent dans les fiches.
} 
langues au XXIe siecle : methodes alternatives et nouveaux dispositifs d'accompagnement, GRAMM-R. Études de linguistique francaise, p. 17-33. Bruxelles, Peter Lang.,

Gyroux L., (2016). La place et le(s) role(s) de la langue maternelle des apprenants en cours de langue etrangere. Synergies France, 10, 55-68. En ligne : https ://gerflint.fr/Base/France10/france10.html

MoORE, D., \& CASTELLOTTI, V. (Eds.) (2008). La compétence plurilingue : regards francophones. Transversales, Langues, sociétés, cultures et apprentissages, vol. 23, Peter Lang.

MIRIADI : Mutualisation et Innovation pour un Réseau de l'Intercompréhension à Distance. En ligne : https ://www.miriadi.net/

OlLIVIER, CH. (2007). Dimensions linguistique et extralinguistique de l'intercompréhension. Pour une didactique de l'intercompréhension au-delà des familles de langues. In CAPUCHO, F., MARTINS, A. A. P., DegachHe, C., \& Tost, M. (Eds.) Dialogos em Intercomprenensa, p. 59-74. Lisboa, Universidade Catolica Editora, Lisboa.

REFIC : Référentiel de compétences de communication plurilingue en intercompréhension. En ligne : https ://www.miriadi.net/refic

REFDIC : Référentiel de compétences en didactique de l'intercompréhension. En ligne : https :// www.miriadi.net/refdic

SHEEREN, H. (2016). L'intercompréhension : un nouveau souffle pour les langues romanes minoritaires et pour les dialectes? Lengas, 79. En ligne : https ://journals.openedition.org/lengas/1060

TRoncy, C. (Ed.), (2014), Didactique du plurilinguisme. Approche plurielle des langues et des cultures. Autour de Michel Candelier. Rennes, Presses Universitaires de Rennes (Collection «Des sociétés»).

\section{Auteurs}

Annick Englebert, L’Université Libre de Bruxelles, e-mail : annick.englebert@ulb.ac.be Docteure en philologie romane, agrégée de linguistique française, est professeure d'histoire de la langue française à la Faculté de Lettres de l'Université Libre de Bruxelles, où elle a pendant longtemps organisé l'accueil des étudiants internationaux et où elle a initié entre autres un projet de tandems linguistiques. Elle participe également à des projets de renforcement des capacités linguistiques des enseignants dans différents pays d'Afrique - Congo, Côte-d'Ivoire, Bénin, Mali et, principalement, Sénégal. Technopédagogue, elle développe de nombreux outils d'apprentissage en ligne.

Sabina Gola, L'Université Libre de Bruxelles, e-mail : sabina.gola@ulb.be Docteur en Philosophie et Lettres de l'Université de Liège et, en qualité de Maître de conférences, enseigne langue, linguistique et culture italiennes à l'Université Libre de Bruxelles (ULB). Elle est coordinatrice académique du département de FLE (Français Langue étrangère) et responsable du volet «multilinguisme» dans le cadre de CIVIS (Alliance universitaire européenne, https ://civis.eu/fr). Elle étudie les stratégies d'apprentissage de l'italien par des étudiants francophones et est la conceptrice de la plate-forme MultiGram (grammaire contrastive et communicative multilingue http ://multigram.ulb.ac.be) et la cocréatrice de Roma-Net, plate-forme consacrée à l'intercompréhension des langues romanes (http ://romanet.ulb.be). 